\title{
Effects of Different Types of Boredom on Foreign Language Learning
}

\author{
Hua Wang, \\ Shanghai International Studies University, Shanghai, China \\ Yong $\mathrm{Xu}$ \\ Shanghai International Studies University, Shanghai, China
}

\begin{abstract}
Although boredom is regarded as a unique emotion in foreign language learning (FLL), studies on the effect of different types of boredom on FLL have not been found. This study aimed to fill this gap by examining it in college students in China. Participants $(N=314)$ learned firstly and then took an immediate test and a delayed one after completing Boredom Tendency Scale (BPS) and the Multidimensional State Boredom Scale (MSBS) questionnaire. And the relationship between these two types of boredom (trait boredom; state boredom) and the numbers of words forgotten as the indicator of the effect of FLL was analyzed, the results showed that these two kinds of boredom had interacted, state boredom had main effect on foreign language learning, while trait boredom had no significant effect on it. This study provides initial support to understanding of the effect of different types of boredom on FLL and suggests that the influence of boredom should not be ignored in foreign language teaching.
\end{abstract}

Index Terms - trait boredom, state boredom, numbers of words forgotten, FLL, effect

\section{INTRODUCTION}

Boredom is a common emotion that affects all aspects of people's daily life. Boredom can be defined as a form of disgust which makes subjects be unable to engage in activities that satisfy them (Eastwood, Frischen, \& Fenske 2012). In the field of education, boredom has been regarded as a meaningful psychological-physiological response to educational events, no longer an unnoticed "silence" (Pekrun 2010). The classification of boredom into state boredom and trait boredom based on time and context is the most widely accepted. State boredom is also called situational boredom, which is regarded as an environmental product. It is more determined by external situational factors (such as lack of stimulation). And it is manifested in the short boring experience of individuals in a particular situation (Belton, Priyadharshini 2007; Musharbash 2007). While trait boredom as a personality trait, is regarded as the individual's boring tendency or boredom proneness, more determined by the individual's internal psychological characteristics (such as cognition and motivation). It is considered to be an individual stable boring tendency in different time and different situations (Neu 1998; Farmer \& Sundberg 1986).

Among the studies on the relationship between affective factors and foreign language learning (FLL), Krashen's affective filtering hypothesis (1982) assumes that affective filtering is a psychological barrier that prevents language learners from fully absorbing comprehensible inputs. It can be said that affective factors determine the proportion of input and reception of FLL learners. At present, a large number of studies have confirmed that there is a significant negative correlation between anxiety and second language learning(L2) and FLL (Shao, Yu, Ji 2013; Dewaele, Witney, \& Saito et al. 2017). However, there are rich affective factors, such as happiness, envy, surprise, gratitude, jealousy, shame, boredom and so on in language environments (Swain \& Merrill 2013). Specially, researches of boredom have gradually moved from the edge of emotional research to the mainstream among them. According to Pekrun's control-value theory (2010), boredom is generally described as a willingness to avoid because avoidance and intrinsic motivation are often negatively correlated. Importantly, boredom weakens individual participation and leads to lack of attention. Consequently, boredom reduces the use of cognitive and learning strategies in educational settings. As a negative emotion, can boredom predict the effect of FLL like anxiety? And, do different types of boredom have the same effect on FLL? What is the psychological mechanism of boredom affecting FLL? Studies on the above questions are still scarce. The aim of this study is to fill the gap by examining the effect of different types of boredom on FLL in college students in China. The current study can not only enrich the theoretical construction of L2/foreign language affective factors, but also provide reference in the practice of L2 /foreign language teaching. Therefore, we assume that: (a) the FLL effect of the subjects in low trait boredom will be better than that of those high in trait boredom. In other words, in this study the number of words forgotten of the subjects who are high-trait bored will be larger than that of those who are low-trait bored. In the same way, (b) compared with the subjects in high state boredom, the FLL effect of the subjects low in state boredom will be better. That is to say, in this study the number of words forgotten of the subjects who are high-state bored will be larger than that of those who are low-state bored. (c) And there is an interaction between trait boredom and state boredom on the effect of FLL. To investigate the effects of trait boredom 
and state boredom on FLL, scales associated with boredom were used to investigate the degree of subjects' trait boredom and state boredom. And the number of words forgotten is looked as the indicator of FLL effect, because the effect of learning can be reflected in memory results (Shen Zheng et al. 2010). This study will start with foreign language vocabulary learning for the following reasons: first, direct vocabulary learning is an effective supplement to indirect learning (Sokmen 1997); second, the current teaching environment in China, most students do not agree to rote learning in the aspect of subjective consciousness, but which still accounts for a large component in practice (Wu Xia, Wang Qiang 1998).

\section{RESEARCH METHOD}

\section{A. Experimental Design}

2(high-trait boredom / low-trait boredom) $\times 2$ ( high-state boredom / low-state boredom) was designed. The dependent variable is the number of words forgotten including Chinese words forgotten and non-words forgotten. And the effect of FLL is defined as the result of immediate score in immediate test minus delayed score in delayed test (the number of words forgotten $=$ score $_{\text {immediate }}-$ score $\left._{\text {delayed }}\right)$. The bigger the number is, the worse the FLL effect will be; conversely, the smaller the number is, the better the learning effect will be. The purpose of using a delayed test as a retest is to get a relatively stable learning effect.

\section{B. Subjects}

314 valid questionnaires, 137 males and 177 females, Chinese college students who were healthy, with an average age of $20.03 \pm 1.34$ years. All subjects volunteered to participate in this experiment.

\section{Experimental Materials}

College Students' Boredom Tendency Questionnaire Boredom Tendency Scale (BPS) (Farmer \& Sundberg 1986) is the most mature of trait boredom measurement tools, and Huang Shihua et al. (2010) supplemented and revised the BPS in combination with Chinese cultural background. The scale consists of 30 questions, for example, "I'm very impatient with waiting", "I often find myself with too much time on my hands and nothing to do", etc. It has two dimensions: external stimuli and internal stimuli. External stimuli consist of four factors: monotonicity, loneliness, nervousness, binding and internal stimuli include two factors: self-control and creativity. The 7-point Likert scale method (ranging from $1=$ completely disagree to $5=$ fully agree) is used. The higher the score, the higher the tendency of boredom. This scale has been considered that it has a stable structure validity and has good applicability in the context of Chinese culture. Cronbach's alpha $=0.85$ in this study.

The Multidimensional State Boredom Scale (MSBS) Using the Chinese version of Liu Yong et al. (2013) which was supplemented and revised according to the MSBS (Fahlman et al. 2013) in combination with Chinese cultural background. Total 24 items, for example, "Now I have no patience", "I want to do something interesting, but nothing attracts me". It includes five dimensions of inattention, time perception, low arousal, high arousal and disengagement. Using Likert 7 grade score, from "completely disagree" to "fully agree", in turn recorded as 1 7 points, the higher the total score represents the higher the current state of boredom. The overall $\alpha$ coefficient of the Cronbach of the questionnaire was 0.90 and in this study was 0.79 .

Non-word List Non-words were used as learning materials in this study. The 16 non-words used mainly refer to the non-word materials designed by Gathercole et al. (1991). And the constitution of non-words is controlled according to wordlikeness and the number of syllables. Specific criteria for non-words compiling include: (1) eliminating the non-word that can cause association of real foreign words at sound likeness or morphological likeness etc.; (2) using non-words of monosyllables, disyllables and trisyllables, and keeping the number of vowels and consonants approximately equal. Each non-word is given a Chinese meaning without emotional color, forming 16 foreign-Chinese word pairs. In order to have a more comprehensive understanding of the subjects' knowledge of the two aspects of comprehension and output, the meaning and spelling of the learned non-words were tested. So, in the test stage, 16 pairs of words were divided into two groups on average, half of them were used to investigate the students' memory of Chinese word meaning, and the other half was used to investigate the memory of spelling of foreign language vocabulary. The non-words are as follows:

TABLE 1.

NON-WORD LIST

\begin{tabular}{|c|c|c|c|c|c|c|c|}
\hline $\begin{array}{l}\text { Non-word } \\
\text { languages }\end{array}$ & Chinese & Non-word & Chinese & $\begin{array}{l}\text { Non-wordd } \\
\text { languages }\end{array}$ & Chinese & $\begin{array}{l}\text { Non-word } \\
\text { languages }\end{array}$ & Chinese \\
\hline thicult & 时间 & bidt & 坚硬 & hond & 早 & jis & 硬件 \\
\hline viulu & 下午 & deppelate & 大的 & glitow & 飞船 & bannow & 孩子 \\
\hline blonter & 变成 & tuwhep & 道路 & soku & 商店 & bomme & 储存 \\
\hline mef & 经历 & prindle & 告诉 & ganner & 工作 & glisterin & 明白 \\
\hline
\end{tabular}

\section{Experimental Process}


The specific experimental tasks are as follows:

(1) Questionnaire completion. The subjects completed the survey of Boredom Tendency Scale (BPS) and the Multidimensional State Boredom Scale (MSBS).

(2) Learning stage. After completing the questionnaires, the non-words were learned for 15 minutes.

(3) Testing phase. After the completion of the learning stage, the immediate test is carried out for 10 minutes. After the immediate test, the subjects were arranged to read freely for half an hour and then to carry out the delayed test. The measuring tool is cued recall, that is, the subjects are required to write the corresponding Chinese meaning or non-words as foreign language words form according to the given non-words form or Chinese meaning respectively. The subjects got 1 point for the one correct answer and there was a full score of 16 points.

\section{E. Statistical Data Analysis Spss22.0 Statistical Software Was Used for Data Collation and Analysis}

\section{RESEARCH RESULTS}

(1) Difference tests for additional variables

In order to control the influence of additional variables on dependent variables, one-way ANOVA was carried out for additional variables including anxiety, levels of foreign language before the experiment. There was no significant difference among the 4 groups ( $p>0.05$ ). Based on this, the above variables need not be considered as covariables in the data processing process.

(2) Groups of trait boredom and state boredom

The data were sorted according to the order of college students' Boredom Tendency Scale (BPQ) total scores from high to low, the top $27 \%$ was classified as high-trait boredom group (BPQ total score > 100), and the bottom $27 \%$ as low-trait boredom group (BPQ total score < 83). The average score of BPQ of high-trait boredom group was significantly higher than that of low-trait boredom group, $\mathrm{p}<.001$. For this part of the subjects, according to their corresponding scores of state boredom, high and low state boredom groups were divided, the results showed that the average score of high-state boredom group (MSBS total score > 94) were significantly higher than that of low state boredom group (MSBS total score < 69), p <.001. A total of 170 subjects, including 85 subjects with low-trait boredom ( 40 males and 45 females) and 85 subjects with high-trait boredom ( 40 males and 45 females). Among them, there were 85 subjects in high-state boredom and 85 subjects in low-state boredom respectively as shown in Table 2 and Table 3 below.

TABLE 2.

THE DIFFERENCE TEST OF BPQ SCORE IN HIGH- AND LOW-TRAIT BOREDOM GROUPS

\begin{tabular}{|c|c|c|c|c|c|c|c|}
\hline \multirow[b]{3}{*}{ BPQ } & \multicolumn{2}{|c|}{$\begin{array}{l}\text { Low-Trait Boredom Groups } \\
(\mathrm{n}=85)\end{array}$} & \multicolumn{2}{|c|}{$\begin{array}{l}\text { High -Trait Boredom Groups } \\
(\mathrm{n}=85)\end{array}$} & \multirow[b]{2}{*}{$t$} & \multirow[b]{2}{*}{$p$} & \multirow[b]{2}{*}{$d$} \\
\hline & $\mathrm{M}$ & SD & $M$ & SD & & & \\
\hline & 74.177 & 7.753 & 108.118 & 7.328 & -29.331 & $<.001$ & 4.499 \\
\hline
\end{tabular}

TABLE 3.

THE DifFERENCE TEST OF MSBS SCORE IN HIGH- AND LOW- STATE BOREDOM GROUPS

\begin{tabular}{|c|c|c|c|c|c|c|c|}
\hline \multirow[b]{3}{*}{ MSBS } & \multicolumn{2}{|c|}{$\begin{array}{l}\text { Low-State Boredom Groups } \\
(\mathrm{n}=85)\end{array}$} & \multicolumn{2}{|c|}{$\begin{array}{l}\text { High-State Boredom Groups } \\
(\mathrm{n}=85)\end{array}$} & \multirow[b]{2}{*}{$t$} & \multirow[b]{2}{*}{$p$} & \multirow[b]{2}{*}{$d$} \\
\hline & $\mathrm{M}$ & SD & $\mathrm{M}$ & SD & & & \\
\hline & 60.306 & 14.376 & 98.788 & 14.095 & -17.622 & $<.001$ & 2.703 \\
\hline
\end{tabular}

(3) Effect of trait boredom and state boredom on the number of forgotten words

With trait boredom and state boredom as independent variables, the number of forgotten words was dependent variable, $2 \times 2$ ANOVA. It turned out that the number of forgotten words of high-trait group $(\mathrm{M}=2.88, \mathrm{SD}=2.27)$, higher than that of low-trait group $(\mathrm{M}=2.85, \mathrm{SD}=2.44)$. The main effect of trait boredom is not significant, $\mathrm{F}(1,166)$ $=0.138, \mathrm{p}=0.711$. The number of forgotten words of high-state group $(\mathrm{M}=3.62, \mathrm{SD}=2.53)$ was higher than that of low-state group $(\mathrm{M}=2.11, \mathrm{SD}=1.88)$. The main effect of state boredom is significant, $\mathrm{F}(1,166)=20.067, \mathrm{p}<.001$. The interaction between trait boredom group and state boredom group on FLL is significant, F $(1,166)=4.156, p<$ 0.05. A further simple effect analysis found that the number of forgotten words of high-state group in low trait boredom $(\mathrm{M}=4.08, \mathrm{SD}=2.58)$ was significantly higher than that of low-state group in low trait boredom $(\mathrm{M}=1.85, \mathrm{SD}=1.81)$, $\mathrm{F}(1,166)=21.244, \mathrm{p}<.001$. And compared with the number of forgotten words of low-state group in high trait boredom $(\mathrm{M}=2.42, \mathrm{SD}=1.95)$, that of forgotten words of high-state group with in high trait boredom $(\mathrm{M}=3.26, \mathrm{SD}=$ $2.45)$, there was no significant difference between them, $\mathrm{F}(1,166)=2.979, \mathrm{p}=0$. 086 . The interaction between trait boredom and state boredom on the number of forgotten words is shown in Fig1. 


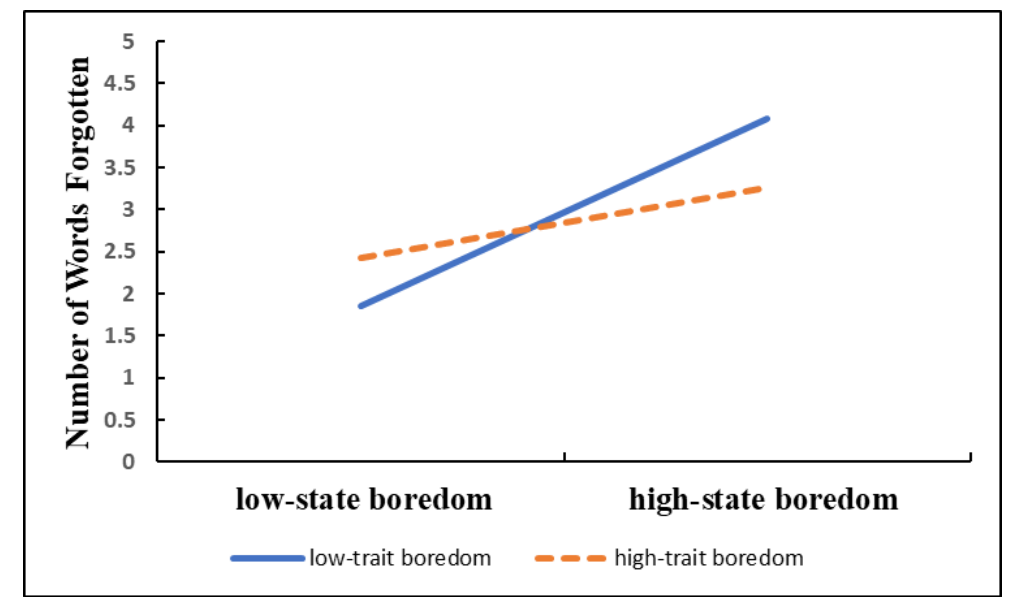

Fig .1 Interaction between Trait Boredom and State Boredom on the Number of Forgotten Words Note: Number of words forgotten means the results of subjects' immediate scores minus delayed scores, it was considered as the indicator reflecting the effect of FLL. (Explained in above.)

\section{DISCUSSION}

(1) The effect of trait boredom on FLL

That there was no significant difference in words forgotten between high-trait group and low- trait boredom group in this study, which is not in line with the research hypothesis (a). The possible explanation is that as a personality trait, trait boredom describes the individual's boredom sensitivity in different situations rather than the individual's ability to cope with boredom (Mercer-Lynn, Bar, \& Eastwood 2014). In other words, trait boredom reflects the ability of individuals to monitor themselves rather than regulate themselves. Individuals with high trait boredom have a high sensitivity to boredom, but they are not necessarily better at coping with boredom by regulating their own behavior. There may be no significant difference in the ability to regulate boredom between the high-trait boredom group and the low-trait boredom group, so high-trait boredom group and the low-trait boredom group did not show significant difference in foreign language learning effect.

(2) Effect of state boredom on FLL

Compared with subjects in low state boredom situations, those in high state boredom tended to forget more in this study. This is consistent with hypothesis (b). And it is also consistent with the results of previous studies. On the one hand, as a unique negative emotion, a typical feature of boredom is a repugnant sense of self (Eastwood, Frischen, Fenske, \& Smilek 2012). And the negative emotions of learners hinder the effectiveness of FLL, because they are obstacles which interfere with learners' learning motivation and self-confidence. The higher the level of boredom, the more obvious the negative feelings of impatience, and then individual will take an evasive attitude in learning activities. Consequently, high-state boredom led to worse learning effects. Krashen's affective filtering hypothesis also supports this view. On the other hand, from the perspective of cognitive processing, attention control is an important component of executive function. And lack of attention is an important feature of state boredom, attention loss affects learners' conscious control of their own thoughts and actions. The higher the state boredom, the less attention. And cognitive failure occurs more frequently when individuals lack of attention management. In other words, state boredom affects learning strategies and self-regulation by reducing the use of cognitive and metacognitive learning strategies. Specifically, state boredom makes individuals reduce the use of deep learning strategies.

(3) The interaction between state boredom and trait boredom on foreign language learning

It was found that the number of forgotten words of high-state boredom group was significantly higher than that of low-state boredom group in low trait boredom group, while there was no significant difference between them in high trait boredom group. The results show that there was an interaction between state boredom and trait boredom on FLL, which is in accordance with hypothesis (c). In low trait boredom group, the number of words forgotten of the high-state boredom group significantly larger than that of high-state boredom group, this suggests that state boredom regulates the relationship between low-trait boredom and the amount of forgetting. While in high trait boredom group, the change of state boredom did not bring about a significant difference in the number of forgotten words. This indicates that state boredom does not regulate the relationship between high-trait boredom and learning effects. Boredom is an emotion characterized by a lack of meaning and challenge (Fahlman et al. 2009), and it has the function of self-regulation (Van, Igou, \& Sedikides 2013). Van, Igou, \& Sedikides (2013) think boredom is closely related to two self-regulating goals. One is that boredom makes individuals seek for stimulus. Second, boredom makes people maintain a sense of the meaning of their own behavior. From the relationship between boredom and self-regulation, different learning effects in different state boredom situations can be understood better. In low trait boredom group, individuals experience low frequency and low intensity of boredom, once you experience high levels of state boredom, it will inspire them to put more effort into self-regulation. And in this situation, learning can provide a sense of meaning. On the contrary, in high trait boredom group, high trait boredom is a recurring pattern, individuals are accustomed to high-intensity boredom. 
Individuals are not willing to make more efforts to change their current meaningless state, even in high state boring situations. They will maintain the current situation without regulating themselves positively. This means that to high-trait boredom group, the ability of self-regulation is weak, even in high state boring situations. According to Pekrun's control-value theory, compared with those in high trait boredom group, the individuals in low trait boredom group, especially those with low-state boredom can seek the meaning of the current task and participate in it more actively. As a result, their cognitive and behavioral performance is better than that of any other group.

\section{CONCLUSIONS}

This paper focused on the influence of two different types of boredom, trait boredom and state boredom, on the effect of FLL. Compared with the number of the forgotten words of subjects which reflecting the effect of FLL as the indicator, it was found that the effect of trait boredom on FLL was not significant, while the effect of state boredom had the main effect on FLL. And there was an interaction between state boredom and trait boredom on FLL. The number of the forgotten words of those with low-state boredom in low trait boredom group was relatively lower than any other group. In short, the effect of FLL of this group was better than any other group. Like anxiety, boredom also has a negative effect on FLL. Therefore, boredom, specially, state boredom is also a negative affective factor that cannot be ignored in FLL. This study, the effect of boredom on FLL, can not only enrich the theoretical construction of foreign language affective factors, but also provide reference for foreign language teaching practice. We can not ignore the influence of boredom on the effect of FLL in educational settings. Teachers should actively improve teaching methods, set up meaningful and challenging learning tasks, awaken students' attention (Pekrun et al. 2010), and use boredom regulation mechanism (Sandi 2016) to stimulate students' interest and buffer the influence of negative emotion on academic achievement (Fritea 2013), so as to improve the effect of FLL. This study provides initial support to test effect of different types of boredom on FLL, the research in this area is still preliminary, which needs to be validated by more scientific research paradigms to explain the neural mechanisms underlying behavioral performance. In the future, it's necessary to understand cognition and behaviors elicited by boredom in a multi-modal way. For example, event-related potentials (ERP), functional magnetic resonance imaging (fMRI) and other techniques can be used to examine brain region activation patterns at different levels of boredom. Further, it is possible to clarify the cognitive characteristics at all stages of boredom formation and development, and to systematically elucidate the psychological and physiological mechanisms of the effect of boredom on FLL.

\section{ACKNOWLEDGMENTS}

This work was supported in part by a grant from the school-level major project of Shanghai International Studies University "Research on the Neural Mechanism of Multilingual Learning" (2017114005).

Note This work is the periodical research result of "Negative Emotion and Language Learning: the Influence of Boredom on Foreign Language Learning" (41003621), an academic guidance project of Shanghai International Studies University.

\section{REFERENCES}

[1] Belton T, Priyadharshini E. (2007). Boredom and schooling: a cross-disciplinary exploration. Cambridge Journal of Education, (37), 579-595.

[2] Dewaele J M, Witney J, Saito K, et al. (2017). Foreign language enjoyment and anxiety: the effect of teacher and learner variables. Language Teaching Research, 22 (6): 676-697.

[3] Eastwood JD, Frischen A, Fenske MJ, et al. (2012). The Unengaged Mind Defining Boredom in Terms of Attention. Perspect. Psychol Sci, 7 (5), 482-495.

[4] Fahlman, Shelley, A., Mercer, Kimberley, B. et al. (2009). Does a lack of life meaning cause boredom? results from psychometric, longitudinal, and experimental analyses. Social \& Clinical Psychology, 28, 3, 307-340.

[5] Fahlman, S. A., Mercer-Lynn, K. B., Flora,D. B. \&Eastwood, J. D. (2013). Development and validation of the multidimensional state boredom scale. Assessment, 20(1), 68-85.

[6] Farmer R, Sundberg N. (1986). Boredom proneness: The development and correlates of a new scale. Social Issues, (59): 103-119.

[7] Fritea ,I., Fritea, R. (2013). Can motivational regulation counteract the effects of boredom on academic achievement? Procedia-Social and Behavioral Sciences, 78: 135-139.

[8] Gathercole S E, Wdlis C, Emslie H, et al. (1991). The Influences of Number of Syllables and Wordlikeness on Children's Repetition of Nonwords. Applied Psycholinguistics, (12), 349-367.

[9] Huang Shihua, Li Dongling, Zhang Wei, et al. (2010). The Preliminary Compilation of College Students' Boredom Tendency Questionnaire. Psychological Development and Education, 26(3), 308-314.

[10] Krashen S D. (1982). Principles and Practice in Second Language Acquisition. New York: Pergamon Press.

[11] Liu Yong, Chen Jianzhi, Jiang Meng, et al. (2013). Application of Chinese Version of Multidimensional State Boredom Scale in Chinese college Students. Chinese Journal of Clinical Psychology, (4), 558-561+540.

[12] Mercer-Lynn, K. B., Bar, R. J., \& Eastwood, J. D. (2014). Causes of Boredom: the Person, the Situation, or both? Personality \& Individual Differences, 56, 122-126. 
[13] Musharbash Y. (2007). Boredom, time, and modernity: An example from aboriginal Australia. American Anthropologist, (109), 307-317.

[14] Neu J. (1998). Boring from within: endogenous Vs reactive boredom. In Emotions in Psychopathology: Theory and Research. London: Oxford University Press, 158-170.

[15] Pekrun R. T, Goetz L M, Daniels R H, et al. (2010). Boredom in achievement settings: Exploring control-value antecedents and performance outcomes of a neglected emotion. Educational Psychology, (102), 531-549.

[16] Sandi, M. (2016). The Upside of Downtime: Why Boredom is Good. United Kingdom: Robinson Publishing

[17] Shen Zheng, Fang Fang, Yang Jiongjiong, et al. (2010). Introduction to Cognitive Neuroscience. Beijing: Peking University Press

[18] Sokmen, A. (1997). Current Trends in Teaching Second Language Vocabulary. In N. Schmitt, \& M. McCarthy (Eds.), Vocabulary: Description, Acquisition and Pedagogy. Cambridge: Cambridge University Press, 237-257.

[19] Shao K, Yu W, Ji Z. (2013). An exploration of Chinese EFL students' emotional intelligence and foreign language anxiety. Modern Language Journal, 97 (4), 917-929.

[20] Swain, Merrill. (2013). The inseparability of cognition and emotion in second language learning. Language Teaching, 46 (02), $1-13$.

[21] Van Tilburg, W. A. P., Igou, E. R., \& Sedikides, C. (2013). In Search of Meaningfulness: Nostalgia as an Antidote to Boredom. Emotion, 13(3), 450-461.

[22] Wu Xia, Wang Qiang. (1998). Vocabulary Learning Strategies for Non-English majors. Foreign Language Teaching and Research, (01), 55-59.

Hua Wang (First author) was born in Jiangsu, China in 1979. She is currently a PH.D. student in Shanghai International Studies University.

Her research interests include psycholinguistics, cognitive neurolinguistics, second language acquisition and English teaching method.

Yong Xu (Corresponding author) was born in Anhui, China in 1970. He received his PH.D. degree in applied linguistics from Shanghai International Studies University in 2006.

$\mathrm{He}$ is currently an associate professor in School of International Business Administration, Shanghai International Studies University. His research interests include psycholinguistics, cognitive neurolinguistics, English teaching method and language testing.

Dr. Xu is a member of Chinese Cognitive Neurolinguistics Research Association, is also a member of the expert group of CET-4 and CET-8. 